\title{
Acesso a medicamentos de alto preço: desigualdades na organização e resultados entre estados brasileiros
}

\author{
Access to high-priced medicines: inequalities \\ in the organization and the results among Brazilian states
}

\author{
Marina Raijche Mattozo Rover (https://orcid.org/0000-0002-2123-4493) ${ }^{1}$ \\ Emília Baierle Faraco (https://orcid.org/0000-0002-9388-353X) ${ }^{2}$ \\ Claudia Marcela Vargas-Peláez (https://orcid.org/0000-0001-5011-8718) ${ }^{3}$ \\ Claudia Flemming Colussi (https://orcid.org/0000-0002-3395-9125) ${ }^{1}$ \\ Sílvia Storpirtis (https://orcid.org/0000-0001-6156-6890) ${ }^{4}$ \\ Mareni Rocha Farias (https://orcid.org/0000-0002-4319-9318) ${ }^{1}$ \\ Silvana Nair Leite (https://orcid.org/0000-0002-5258-9684) ${ }^{1}$
}

${ }^{1}$ Departamento de Ciências Farmacêuticas, Centro de Ciências da Saúde, Universidade Federal de Santa Catarina. R. Delfino Conti S/N, Trindade, 88040370. Florianópolis SC Brasil. marinarover@yahoo.com.br ${ }^{2}$ Grupo de Pesquisa em Políticas e Serviços Farmacêuticos, Universidade Federal de Santa Catarina.

Florianópolis SC Brasil.

${ }^{3}$ Fundação IFARMA.

Bogotá Colômbia

${ }^{4}$ Departamento de

Farmácia, Faculdade de

Ciências Farmacêuticas,

Universidade de São Paulo.
Abstract This case study aimed to characterize the Specialized Component of Pharmaceutical Services (CEAF) organization in four Brazilian states from diverse regions of the country. Data were collected with representatives of CEAF management from states in different regions, who answered a 21-question questionnaire on scope, organization, financing, hurdles, and facilitators. This information was complemented with data from national health surveys, DataSUS, the applied resources, and socioeconomic indicators. Differences were observed between states on issues such as the proportion of users and the decentralization of services. These characteristics seem to be related to the level of development concerning the socioeconomic indicators used. Advances in access to medicines were highlighted, despite the difficulties complying with the CEAF's objectives, such as insufficient resources, the qualification of human resources, and the provision of necessary visits and exams. The results point to advances, different forms of organization and highlight the need for more in-depth studies on the clinical and economic outcomes achieved as a strategy to outline solutions to achieve the comprehensive and equal care for users.

Key words Pharmaceutical care, Access to health services, High-cost technology, Essential medicines
Resumo Este estudo de caso visou caracterizar a organização do Componente Especializado da Assistência Farmacêutica (CEAF) em quatro estados, de diferentes regiões do país. A coleta de dados foi realizada junto a representantes da gestão do CEAF, os quais responderam um questionário com 20 perguntas sobre: abrangência, organização, financiamento, barreiras e facilitadores. Essas informações foram complementadas com dados de inquéritos nacionais de saúde, do DataSUS, os valores investidos e indicadores socioeconômicos. Observaram-se diferenças entre os estados em questões como a proporção de usuários e a descentralização dos serviços. Estas características parecem estar relacionadas com o grau de desenvolvimento em termos dos indicadores socioeconômicos utilizados. Destacaram-se avanços no acesso a medicamentos, apesar das dificuldades para o cumprimento dos objetivos do CEAF, como a insuficiência de recursos, de qualificação da força de trabalho e da oferta de consultas e exames necessários. Os resultados indicam avanços, diferentes formas de organização e destacam a necessidade de estudos mais aprofundados relativos aos resultados clínicos e econômicos alcançados, como uma estratégia para traçar soluções para o atendimento integral e equânime dos usuários.

Palavras-chave Assistência farmacêutica, Acesso aos serviços de saúde, Tecnologia de alto custo, Medicamentos essenciais 


\section{Introdução}

Os medicamentos têm importante papel na atenção à saúde. No entanto, os custos crescentes das alternativas terapêuticas incorporadas nos sistemas de saúde exigem estratégias para garantir o acesso e a integralidade dos tratamentos. Este aspecto tem preocupado pesquisadores e gestores dos sistemas de saúde, inclusive nos países desenvolvidos $^{1-6}$.

Para garantir o acesso e o uso racional dos medicamentos (URM), o Brasil tem implementado diferentes políticas públicas ${ }^{7-9}$. Entre as estratégias para a ampliação do acesso, o Componente Especializado da Assistência Farmacêutica (CEAF) foi implantado visando a integralidade dos tratamentos, a partir de linhas de cuidado definidas em Protocolos Clínicos e Diretrizes Terapêuticas (PCDT/MS), os quais definem não só o algoritmo e tratamento, mas também os critérios de diagnóstico e os mecanismos para o monitoramento clínico ${ }^{8,9}$.Ainda que o Brasil não adote um parâmetro para definir medicamentos considerados de alto custo (como acontece no sistema de saúde Inglês) ou de alto preço $^{10}$, é no CEAF que são disponibilizados os medicamentos de uso ambulatorial de maior preço médio no SUS, incluindo os mais recentemente incorporados pela CONITEC como o ecolizumabe ${ }^{11}$.

$\mathrm{O}$ uso dos termos "medicamentos de alto custo" ou "alto preço" ainda não foi definido internacionalmente e pode variar no mesmo país ${ }^{12}$. Alguns países categorizam os medicamentos de alto custo pelo preço limite por paciente ou por ano a ser reembolsado pelo pagador público. Esses medicamentos geralmente apresentam patente de monopólio e representam uma carga financeira significativa para o sistema público de assistência médica e/ou maiores despesas diretas para pessoas físicas ${ }^{13}$.

$\mathrm{O}$ financiamento dos medicamentos do CEAF é tripartite, entretanto aqueles indicados para doenças de maior complexidade, de elevado impacto financeiro e que se incluem em ações do Complexo Industrial da Saúde, são adquiridos de forma centralizada pelo MS, ou financiados por este, por meio da transferência de recursos aos estados $^{8,9}$.

Seguindo os princípios de gestão do SUS, as etapas que compõem a execução do CEAF são descentralizadas, e as Secretarias Estaduais de Saúde (SES) são responsáveis pela organização dos serviços para atendimento à população, incluindo a dispensação dos medicamentos. Contudo, algumas atividades podem ser realizadas pela rede de serviços públicos dos municípios, desde que ocorra a pactuação entre os gestores ${ }^{9}$.

Neste contexto, Rover e colaboradores ${ }^{14-16}$ destacam que a gestão do CEAF pode ser influenciada por diversos fatores, e que a garantia da integralidade dos tratamentos envolve, além do acesso ao medicamento, acesso a outros cuidados e serviços de saúde, e a articulação entre estes $^{14}$. Na realidade, segundo os autores a falta de inter-relação da Assistência Farmacêutica com outros setores da saúde, que se traduz, por exemplo, no descompasso entre a demanda de serviços relacionada ao CEAF e a oferta destes pelo estado resulta na fragmentação do cuidado ${ }^{15,16}$.

Desde a implementação do CEAF, os investimentos da União para o financiamento do Componente vêmcrescendo ${ }^{1,17,18}$, porém poucos estudos foram realizados em relação a sua organização e gestão, aos resultados alcançados e sobre sua abrangência nos diferentes estados e regiões brasileiras, considerando as desigualdades conhecidas.

Assim, considerando as diferenças entre as estruturas da assistência farmacêutica no país, questiona-se quais são os aspectos que ainda necessitam ser aprimorados para o alcance dos objetivos do CEAF. Nesse sentido, o objetivo deste estudo foi a caracterização das diferentes formas de organização, gestão e do acesso aos medicamentos do CEAF em quatro estados de diferentes regiões do país e sua relação com indicadores socioeconômicos e de saúde.

\section{Métodos}

Trata-se de um estudo de caso, desenvolvido com dados coletados dos anos de 2014 e 2015, através de questionários e bancos de dados secundários. Os estados participantes foram escolhidos por meio de amostragem por conveniência utilizando como critério a disponibilidade do gestor em responder. Os convites para a participação no estudo foram enviados por e-mail para seis estados e o Distrito Federal. Cinco deles e o Distrito Federal aceitaram participar e, neste estudo, quatro estados foram incluídos por terem apresentados todos os dados necessários. Os gestores participantes responderam os questionários e enviaram por e-mail.

O questionário foi desenvolvido com base nas diretrizes previstas nos PCDT e nas regulamentações do Componente ${ }^{8,9}$, e foi validado através de discussões com o grupo de pesquisa até a obtenção de consenso sobre as questões mais re- 
levantes para o estudo. O questionário consensual consistiu em 20 questões (nove abertas e 11 fechadas) relacionadas à (ao): abrangência (por ex. número de usuários); forma de organização do CEAF (por ex. descentralizada, municipalizada); financiamento (por ex. valores investidos); infraestrutura (por ex. número de unidades, centros de referência - CR/polos de aplicação); serviços logísticos e clínicos (por ex. problemas na aquisição, monitoramento previsto nos PCDT); questões abertas sobre a percepção de facilitadores e fragilidades existentes na gestão do CEAF no estado.

De cada estado foram coletados os seguintes dados: população, Produto Interno Bruto (PIB), Índice de Desenvolvimento Humano Municipal $(\text { IDHM })^{19,20}$ e Índice de Desenvolvimento do SUS (IDSUS $)^{21}$. Os dados de PIB e IDSUS foram categorizados em três grupos de acordo com a ordem decrescente da posição dos estados: terço superior (Estados $1^{\mathrm{a}}$ a $\left.9^{\mathrm{a}}\right)$, terço médio $\left(10^{\mathrm{a}}\right.$ a $\left.18^{\mathrm{a}}\right)$, terço inferior $\left(19^{\mathrm{a}}\right.$ a $27^{\mathrm{a}}$ ). No caso do IDHM foi considerada a classificação:muito baixo: 0-0,499; baixo: 0,500-0,599; médio: 0,600-0,699; alto: 0,7000,$799 ;$ muito alto: $>0,800^{20}$.

Adicionalmente, foram coletados dados dos indicadores de acesso a serviços de saúde e medicamentos da Pesquisa Nacional de Saúde ${ }^{22}$ (percentual da população que acessa a todos medicamentos, da população que acessa a consulta médica e da população com plano privado de saúde) e dados do DataSUS ${ }^{23}(\%$ de recursos aplicados pelos Estados EC29 - em saúde). Ainda foram considerados os valores investidos para o CEAF, pelo MS, em cada um dos estados,segundo as portarias correspondentes ${ }^{24-27}$.

Para a conversão ao dólar dos Estados Unidos foi utilizada a taxa de câmbio reportada pelo Banco Central do Brasil (2,34 reais por dólar).

As respostas às perguntas abertas foram analisadas e categorizadas, seguindo as etapas de tratamento e análise de dados qualitativos de Pope et al. ${ }^{28}$

Este estudo foi aprovado pelo Comitê de Ética em Pesquisa com Seres Humanos da Universidade Federal de Santa Catarina.

\section{Resultados}

Os representantes da gestão participantes da pesquisa eram farmacêuticos, com vínculo estatutário e com dois anos ou mais de experiência no cargo. A população dos quatro estados juntos representava aproximadamente $35 \%$ da população brasileira.
Na Tabela 1 são apresentados o IDHM, o PIB, o IDSUS, e indicadores de acesso aos medicamentos, às consultas e aos planos de saúde. É possível observar que os estados analisados apresentavam diferenças nos indicadores IDHM, IDSUS e PIB, que coincidiam com os dados dos indicadores de acesso a medicamentos e serviços de saúde.

Os estados com maiores PIB, IDHM e IDSUS (Sul e Sudeste) apresentaram, também, maior acesso a medicamentos, consultas e plano privado de saúde.

Em relação especificamente ao CEAF, observaram-se diferenças no financiamento, na proporção de usuários por habitantes e na organização do Componente (descentralização dos locais de dispensação) (Tabelas 2 e 3 ).

As informações sobre o financiamento para aquisição dos medicamentos demonstraram que os maiores valores (absolutos) de recursos são repassados pela União aos estados com maior número de usuários (Sul e Sudeste). Por sua vez, os outros dois estados foram os que apresentaram maior gasto proporcional, no financiamento dos medicamentos.

A proporção de pessoas atendidas pelo CEAF foi maior no estado da região Sul e menor no da região Norte. Destacaram-se, também, diferenças no grau de descentralização do componente (entendida como maior número de locais de dispensação) sendo maior nos estados do Sul e Sudeste. Os dados mostraram maior disponibilidade de polos de aplicação de medicamentos no estado da Região Sudeste.

Segundo a opinião dos participantes, a execução do CEAF apresentava fragilidades em diferentes etapas, tanto nos processos logísticos quanto assistenciais e de articulação com os municípios, como categorizado no Quadro 1. Como exemplo, cita-se a falta de informações sobre o atendimento da primeira linha de cuidado em alguns estados; a demanda reprimida devido a programação da compra centralizada ser feita sem reserva técnica; o longo tempo para avaliação das solicitações; debilidades na oferta de consultas com especialistas e exames (quando exigidos) para acesso aos medicamentos. Ainda, segundo os participantes, havia a necessidade da centralização da compra de outros medicamentos, principalmente, devido ao impacto econômico que representavam e porque os valores repassados pelo MS eram insuficientes.

Entretanto, também foram descritas fortalezas, como a ampliação do acesso aos medicamentos, pela incorporação de novas tecnologias e au- 
Tabela 1. Características contextuais dos estados analisados.

\begin{tabular}{|c|c|c|c|c|}
\hline Indicador & $\begin{array}{l}\text { Estado } \\
\text { (Norte) }\end{array}$ & Estado (Sul) & $\begin{array}{c}\text { Estado } \\
\text { (Nordeste) }\end{array}$ & $\begin{array}{c}\text { Estado } \\
\text { (Sudeste) }\end{array}$ \\
\hline IDHM $^{\mathrm{a}}$ & Médio & Alto & Médio & Alto \\
\hline IDSUS $^{\mathrm{b}}$ & Terço inferior & Terço superior & Terço inferior & Terço superior \\
\hline PIB $(\text { ano })^{\mathrm{c}}$ & Terço médio & Terço superior & Terço inferior & Terço superior \\
\hline 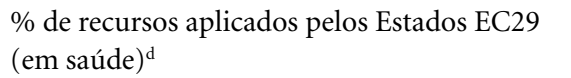 & 13,5 & 11,2 & 13,4 & 12,4 \\
\hline $\begin{array}{l}\text { \% da população que acessa a todos os } \\
\text { medicamentos }^{\mathrm{e}}\end{array}$ & 75,8 & 84,8 & 80,8 & 83,1 \\
\hline $\begin{array}{l}\text { \% da população que acessa a consulta } \\
\text { médica }^{\mathrm{e}}\end{array}$ & 57,5 & 72,8 & 66,4 & 78,4 \\
\hline \% da população com plano privado de saúde & 13,7 & 32,9 & 13,9 & 42,1 \\
\hline
\end{tabular}

Tabela 2. Dados relativos ao financiamento do CEAF nos quatro estados.

\begin{tabular}{|c|c|c|c|c|}
\hline & \multicolumn{4}{|c|}{ Estados } \\
\hline & Norte & Sul & Nordeste & Sudeste \\
\hline $\begin{array}{l}\text { Valor total investido em medicamentos - Estado e União } \\
\left(\text { em milhões }{ }^{\star}\right)\end{array}$ & 21,2 & 128,2 & 28,1 & 890,4 \\
\hline Valor investido pela União em medicamentos (em milhões ${ }^{\star}$ ) & 15,4 & 115,6 & 22,5 & 719,4 \\
\hline$\%$ investido pelo estado em medicamentos & 27 & 9,8 & 19,9 & 19,2 \\
\hline Valor médio investido por usuário* & 3.023 & 1.224 & 2.342 & 1.644 \\
\hline Investimento na qualificação de $\mathrm{RH}^{*}$ & - & - & - & - \\
\hline Investimento na estruturação dos serviços ${ }^{*}$ & 64.000 & 3.418 .000 & - & - \\
\hline
\end{tabular}

Fonte: Dados de investimento da União - Portarias ${ }^{24-27}$, os demais dados foram obtidos nas entrevistas.

*Valores calculados em dólares americanos.

Tabela 3. Dados relativos à cobertura e a organização do CEAF nos quatro estados.

\begin{tabular}{lrrrr}
\hline & \multicolumn{4}{c}{ Estados } \\
\cline { 2 - 5 } & Norte & Sul & Nordeste & Sudeste \\
\hline No usuarios do CEAF/1.000 habitantes & 0,9 & 10 & 3,2 & 13,2 \\
No locais de dispensação/100 municípios & 18 & 75 & 7 & 97 \\
Média de número de usuários por local de dispensação & 269 & 347 & 800 & 869 \\
Número de Centros de Referência/Polos de Aplicação & 0 & $15^{\text {a }}$ & $2^{\text {b }}$ & 142
\end{tabular}

Fonte: $\mathrm{IBGE}^{19}$ (número de habitantes e municípios por estado). Os demais dados foram obtidos nas entrevistas.

aAdministração de Toxina Botulínica, Imiglucerase e medicamentos para o tratamento da Hepatite C. ${ }^{\text {b }}$ Administração de medicamentos para o tratamento da Hepatite C e Esclerose Múltipla.

mento do número das condições clínicas para as quais o setor público contempla o tratamento. As principais fragilidades e fortalezas descritas são apresentadas no Quadro 1.

Cabe destacar que ainda foi pontuada pelos participantes o curto período de renovação das solicitações para as doenças crônicas (trimestral) e a falta de dados relativos aos resultados clínicos e econômicos alcançados.

\section{Discussão}

Pesquisas nacionais têm identificado que o acesso aos medicamentos, incluindo os setores público e privado, tem evoluído de forma consistente no Brasil ${ }^{1,29}$, como resultado das políticas públicas implementadas nos últimos anos ${ }^{8}$. Nesse contexto, os resultados positivos do CEAF relativos à ampliação do elenco, a construção das linhas 
Quadro 1. Principais fragilidades e fortalezas apontadas pelos gestores.

\begin{tabular}{|c|c|}
\hline Fortalezas & Estados \\
\hline Ampliação do acesso aos medicamentos & Norte/Sul/Nordeste/Sudeste \\
\hline Construção das linhas de cuidado & Sul \\
\hline Descentralização da dispensação nos municípios & Sul/Sudeste \\
\hline $\begin{array}{l}\text { Compras centralizadas pelo MS dos medicamentos com maior impacto } \\
\text { financeiro }\end{array}$ & Sul/Sudeste \\
\hline $\begin{array}{l}\text { Presença do avaliador e autorizador em unidades de atendimento, } \\
\text { proporcionando a dispensação imediata }\end{array}$ & Sudeste \\
\hline Sistema de informação gerencial & Sul \\
\hline \multicolumn{2}{|l|}{ Fragilidades } \\
\hline Problemas na aquisição ${ }^{a}$ & Norte/Sul/Nordeste/Sudeste \\
\hline Financiamento insuficiente $^{\mathrm{b}}$ & Norte/Sul/Nordeste/Sudeste \\
\hline Não implementação do monitoramento clínico previsto no PCDT & Norte/Sul/Nordeste \\
\hline Necessidade da centralização da compra de outros medicamentos ${ }^{c}$ & Norte/Sul/Nordeste \\
\hline $\begin{array}{l}\text { Necessidade de atualização periódica dos PCDT e incorporação de outros } \\
\text { medicamentos }^{\mathrm{d}}\end{array}$ & Norte/Sul/ Nordeste/Sudeste \\
\hline Força de trabalho insuficiente & Sul/Nordeste/Sudeste \\
\hline Debilidades na estrutura física & Nordeste \\
\hline Poucas unidades de atendimento (incluindo os CR) & Norte/Nordeste/Sudeste \\
\hline Falta de pactuação com os municípios para a execução do CEAF & Sudeste \\
\hline Falta de padronização das etapas de execução & Sudeste \\
\hline Sistemas informatizados que não interoperam & Norte/Sul/Nordeste/Sudeste \\
\hline \multicolumn{2}{|c|}{ 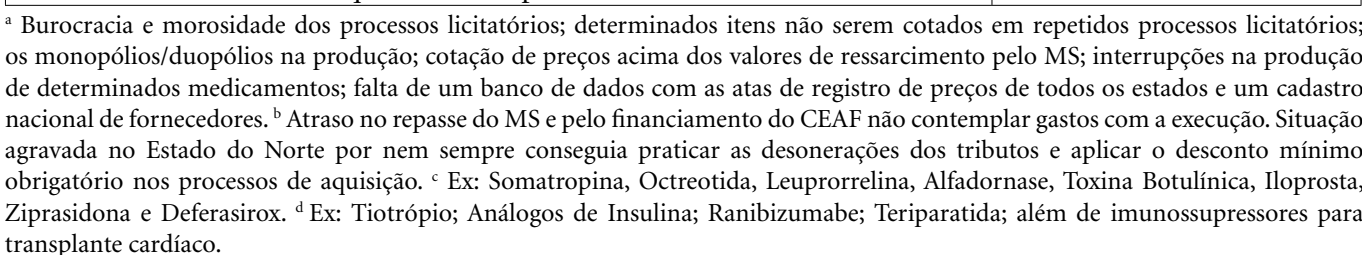 } \\
\hline
\end{tabular}

Fonte: Dados obtidos nas entrevistas.

de cuidado e o aumento do número de usuários atendidos foram destacados pelos participantes como as principais fortalezas. Porém, os achados deste estudo demonstram que os resultados entre os estados não são homogêneos, e que ainda existem dificuldades para a execução plena do Componente.

A heterogeneidade observada, tanto na forma de organização para atendimento dos usuários quanto no número de usuários, se relaciona com as diferenças contextuais dos estados e com a capacidade de gestão, dado que o desempenho dos sistemas de saúde é amplamente influenciado pelas características políticas, socioeconômicas e culturais locais ${ }^{30,31}$. E, por sua vez, o acesso aos medicamentos é altamente dependente da organização e funcionamento dos sistemas de saúde também no nível local ${ }^{32}$.

Nesse sentido, os resultados deste estudo sinalizam que, apesar dos PCDT serem diretrizes nacionais, os estados das regiões Sul e Sudeste apresentavam uma proporção maior de usuários atendidos pelo CEAF, estados com maior disponibilidade de recursos econômicos (maior PIB), de serviços públicos (maior IDSUS) e maior cobertura de planos privados de saúde. A associação inversa entre posição socioeconômica e subutilização de medicamentos foi previamente observada no Brasil e corrobora aos achados de Luz e colaboradores ${ }^{33}$.

Embora para Penchansky e Thomas ${ }^{34}$, a acessibilidade do serviço seja determinada pela relação entre o volume e o tipo de serviço disponível, e volume e tipo de necessidade dos usuários, os resultados encontrados corroboram com a primeira afirmação, mas não permitem afirmar que o menor acesso ao CEAF nas regiões Norte e Nordeste foram resultado de uma menor necessidade, uma vez que não há dados epidemiológicos que indiquem esta hipótese.

Sabe-se também que, embora o Brasil tenha apresentado avanços importantes em relação ao 
acesso aos serviços de saúde, principalmente na atenção básica, as limitações da oferta de serviços de alta e média complexidade que são necessários para o cumprimento de critérios estabelecidos pelos PCDT, continuam sendo um grande desafio para o setor público do SUS ${ }^{1,6,35}$. Estas limitações causam longos tempos de espera, o que resulta na busca por atendimento especializado no setor privado ou pela via judicial, o que onera ainda mais o estado,14-16,36,37. Assim, cria-se um duplo padrão de acesso aos medicamentos do CEAF (entre os que têm e os que não têm condições de acessar consultas e exames no sistema privado), além dos arranjos informais (como os mecanismos para transpor as filas $)^{14}$.

Buendgens e colaboradores ${ }^{38}$ concluíram $^{2}$ que, de todos os usuários com diagnóstico de artrite reumatoide estudados, só um teve acesso a todos os serviços de saúde requeridos para seu tratamento no setor público. Porém, este estudo também demonstrou que mais de 70\% do financiamento do tratamento da doença foi arcado pelo setor público. Assim, mesmo com limitações, o setor público tem arcado com a maior parte do financiamento destes tratamentos.

O presente estudo aponta que a maior proporção de usuários do CEAF se apresenta onde há descentralização ao nível municipal das unidades de dispensação (estados da região Sul e Sudeste), a qual é depende da capacidade de articulação com os municípios e dos recursos disponíveis. Limitações neste sentido já foram demonstradas em estudos anteriores ${ }^{39-41}$, as quais são barreiras para a organização da rede de serviços nos municípios e indicam a necessidade de negociações entre os gestores ${ }^{42}$. Problemas de articulação com os municípios aparecem, também, na não verificação do fornecimento da primeira linha de tratamento, passo fundamental para a garantia da efetividade e integralidade dos tratamentos.

Dado que, como mencionado anteriormente, o acesso aos medicamentos do CEAF está estreitamente relacionado com a disponibilidade de pontos de dispensação e o acesso a outros serviços para o diagnóstico e acompanhamento dos tratamentos ${ }^{14}$, os resultados deste estudo chamam a atenção para a necessidade crescente de coordenação entre os serviços de saúde ${ }^{44}$, considerando a assistência farmacêutica como parte inequívoca da gestão do sistema e não um setor isolado $^{15}$. Os resultados apresentados sugerem que o entendimento real da assistência farmacêutica no planejamento e processo decisório do sistema de saúde é crítico para o alcance de acesso equitativo e oportuno aos tratamentos.
Os resultados mostram, também, diferenças nos percentuais investidos em medicamentos entre os estados. Os valores investidos são relacionados, principalmente, ao número de usuários, o qual está associado ao nível de desenvolvimento e à disponibilidade de serviços de saúde. Em relação aos investimentos do MS para as compras do grupo $1 \mathrm{~B}$, este depende da solicitação dos medicamentos em cada estado, a qual também é determinada pela oferta de serviços. Todavia, a capacidade de gestão na aquisição dos estados também é um fator que influencia a proporcionalidade dos investimentos por usuário. Esta situação é ainda mais crítica no caso dos medicamentos com número de fornecedores limitado, devido aos monopólios gerados pela proteção de patentes. Desta forma, a desoneração dos estados, esperada pela atual pactuação do financiamento dos medicamentos de mais alto preço ${ }^{8,9}$, não é alcançada de forma adequada.

Ainda, os estados que têm menor população e, portanto, menor demanda (Norte e Nordeste) têm uma menor capacidade de negociação frente aos fornecedores, o que significa que os preços de aquisição são maiores, ou seja, a unidade investe proporcionalmente mais para uma mesma condição clínica. Desta forma, os estados, com menores indicadores socioeconômicos e de desenvolvimento do SUS serão os mais onerados.

Apesar das medidas adotadas para redução dos preços de aquisição dos medicamentos, como a definição do Preço Máximo de Venda ao Governo $^{8}$, os resultados apontaram a necessidade do fortalecimento da vigilância sobre o mercado de medicamentos; a implantação de um banco nacional de registro de preços e de fornecedores; bem como a geração de estratégias para a compra conjunta entre os estados, as quais podem contribuir para a regulação dos preços ${ }^{1,45}$. Para Tobar e colaboradores ${ }^{46}$, estas são alternativas para a adoção de políticas centradas no acesso a medicamentos.

Os dados evidenciaram, também, a focalização dos recursos na aquisição dos medicamentos, em detrimento do aprimoramento de outros aspectos relevantes para o alcance do objetivo da integralidade do cuidado, tais como capacitação contínua da força de trabalho e estrutura física adequada. Na perspectiva dos participantes, o fato do repasse financeiro do CEAF ser exclusivo para a aquisição dos medicamentos, onera os estados com os custos da execução, dificultando os investimentos na qualificação dos serviços. Já o estabelecido na regulamentação vigente considera que a execução do CEAF é responsabilidade dos estados ${ }^{9}$. 
Outro aspecto relatado pelos gestores foi o custo adicional do financiamento de medicamentos não contemplados no CEAF, mas que eram fornecidos como resultado de ações judiciais. O fato dos medicamentos solicitados por via judicial, sugeridos para incorporação pelos participantes, já terem sido avaliados pela Comissão Nacional de Incorporação de Tecnologias no SUS e o parecer da maior parte foi desfavorável $^{47-49}$, corrobora a evidência levantada da utilização da judicialização como um mecanismo da indústria farmacêutica para pressionar a incorporação de novas tecnologias no SUS ${ }^{4,50}$. Perante esta situação, surge a necessidade do desenvolvimento de estratégias conjuntas entre o MS e os estados que visem a difusão de informações independentes sobre a eficácia e segurança dos medicamentos, bem como a capacitação de prescritores para URM.

A focalização no produto medicamento também é observada no desenvolvimento das atividades relativas ao CEAF, uma vez que são priorizadas as atividades para a disponibilização dos medicamentos, enquanto a execução das atividades clínico-assistenciais necessárias para o acompanhamento dos tratamentos (p. ex. a implantação dos CR e o monitoramento de acordo com os PCDT) são ainda limitados, fato já registrado por outros estudos ${ }^{1,16,37,51}$. Desta forma, a falta de dados sobre os resultados dos tratamentos resulta na indisponibilidade de informações no que diz respeito à melhoria do estado de saúde da população e efetividade do Componente, o que representa uma fragilidade dos serviços de saúde ${ }^{52}$.

Por fim, os problemas logísticos apontados, somados ao fato de os sistemas informatizados não serem interligados, podem acarretar faltas ou atrasos na disponibilização dos medicamentos. Especificamente em relação aos sistemas de informação, é importante destacar que o repasse financeiro e de medicamentos ocorre somente após transferência de dados ao MS. Falhas e problemas que podem ocorrer nesta transferência, somadas à impossibilidade dos estados solicitarem reservas técnicas para atendimento de novos usuários, podem acarretar a falta ou atrasos nos atendimentos, com impactos negativos individuais e coletivos, pela piora do estado de saúde, uso de terapias e serviços adicionais, e maiores gastos nos tratamentos ${ }^{53,54}$. Sistemas interligados propiciam agilidade no repasse das informações, permitindo $\mathrm{o}$ atendimento de forma oportuna dos usuários e merece especial atenção dos estados e do MS.

Cabe destacar que nos últimos anos parte dos medicamentos demandados pelos participantes deste estudo para aquisição pelo MS tiveram sua compra centralizada e que, mais recentemente, por meio da Portaria no 13 de janeiro de 2020, o MS resolve parcialmente a problemática dos curtos períodos para renovação das solicitações, ao possibilitar que possam ser realizadas para até seis meses de tratamento ${ }^{55}$. Esta medida reduz a burocratização ${ }^{1}$, mas se não adequadamente implementada pode acarretar prejuízos para os pacientes cujas doenças exijam um acompanhamento mais frequente (p. ex. doentes renais crônicos, transplantados, e com doenças inflamatórias ativas).

O estudo teve como limitação a utilização de alguns dados referidos pelos informantes, pois os estados não possuem, ou não disponibilizam publicamente todos os dados relativos à organização e gestão do componente. A amostra foi intencional, desta forma, o estudo não pode ser interpretado como uma avaliação das quatro regiões como um todo. Contudo, os resultados fornecem um panorama das diversas condições apresentadas pelos estados brasileiros e das diferentes condições de acesso aos medicamentos do CEAF.

Esta primeira aproximação ao estudo da organização do CEAF aponta que o componente tem contribuído com a ampliação do acesso a medicamentos de alto preço no Brasil. Porém, dado que o acesso aos medicamentos depende das características contextuais e da capacidade de gestão, os resultados deste estudo evidenciam a necessidade de superar desigualdades entre os estados no acesso aos serviços de saúde, visando o atendimento dos preceitos constitucionais de universalidade $\mathrm{e}$ integralidade do cuidado.

Os resultados sugerem que o país precisa avançar no acesso à terapia medicamentosa garantindo a equidade e oportunidade. Para superar estes desafios, além de medidas relativas à gestão do Componente, é necessária uma maior interação entre os gestores da assistência farmacêutica com os demais gestores do SUS, a fim de desenhar estratégias que visem o fortalecimento do processo de descentralização, e a superação das deficiências na oferta dos serviços especializados.

Observou-se, também, que as diferentes formas de organização e estruturação do CEAF têm impactado no acesso da população aos medicamentos. Preocupa o achado de que os estados com piores indicadores de desenvolvimento são aqueles que investem proporcionalmente mais para uma mesma condição clínica. Por fim, a carência de dados nacionais sobre o CEAF aponta para a necessidade de estudos mais aprofundados relativos aos resultados alcançados, que possibilitem a formulação de políticas públicas visando otimizar a execução do CEAF. 


\section{Colaboradores}

MRM Rover coletou os dados, analisou os achados de campo e redigiu o texto. SN Leite auxiliou na análise dos resultados, na concepção e na redação final. EB Faraco colaborou nas análises dos resultados e na elaboração do texto. MR Farias auxiliou na redação final do texto. CM Vargaz-Pelaez colaborou nas análises dos resultados e na elaboração do texto; C Colussi colaborou na análise dos dados e revisão final do texto. S Storpirtis auxiliou na revisão final do texto.

\section{Agradecimentos}

Este estudo foi financiado em parte pela Coordenação de Aperfeiçoamento de Pessoal de Nível Superior - Brasil (CAPES) - Código Financeiro 001.

\section{Referências}

1. Brasil. Ministério da Saúde (MS). Secretaria de Ciência, Tecnologia e Insumos Estratégicos. Departamento de Assistência Farmacêutica e Insumos Estratégicos. Assistência Farmacêutica no SUS: 20 anos de políticas e propostas para desenvolvimento e qualificação: relatório com análise e recomendações de gestores, especialistas e representantes da sociedade civil organizada [recurso eletrônico]. Brasília: MS; 2018. 125 p.: il.

2. Lu CY, Williams KM, Day RO. The funding and use of high-cost medicines in Australia: the example of anti-rheumatic biological medicines. Aust N Z Health Policy 2007; 4(1):2.

3. Bevan G, Helderman J-K, Wilsford D. Changing choices in health care: implications for equity, efficiency and cost. Health Econ Policy Law 2010;5(3):251-267.

4. Vargas-Peláez CM, Rover MRM, Leite SN, Rossi Buenaventura F, Farias MR. Right to health, essential medicines, and lawsuits for access to medicines - A scoping study. Soc Sci Med 2014; 121:48-55.

5. Fondo Nacional de Recursos. Política y gestión de la cobertura de medicamentos de alto costo. Relevamiento de la experiencia internacional y respuesta del Fondo Nacional de Recursos [Internet]. Primera. Montevideo: FNR; 2010. (Publicación Técnica). [acceso 2020 Abr 25]. Disponible en: http://www.fnr.gub.uy/sites/ default/files/publicaciones/FNR_publicacion_tecnica_13.pdf

6. Paim J, Travassos C, Almeida C, Bahia L, Macinko J. The Brazilian health system: history, advances, and challenges. The Lancet 2011; 377(9779):1778-1797.

7. Brasil. Conselho Nacional de Saúde. Resolução $\mathrm{n}^{\circ}$ 338 de 6 de maio de 2004. Aprova a Politica Nacional de Assistência Farmacêutica [Internet]. maio 6, 2004. [acessado 2020 abr 23]. Disponível em: http://repositorio.ufpe.br/handle/123456789/9696

8. Brasil. Ministério da Saúde (MS). Da Excepcionalidade às Linhas de Cuidado: O Componente Especializado da Assistência Farmacêutica [Internet]. 1 $1^{\mathrm{a}}$ ed. Brasilia: MS; 2010 [acessado 2015 nov 5]. Disponível em: http://bvsms.saude.gov.br/bvs/publicacoes/excepcionalidade_linhas_cuidado_ceaf.pdf

9. Brasil. Ministério da Saúde (MS). Portaria de Consolidação $\mathrm{n}^{\circ} 2$ de 28 de setembro de 2017. Consolidação das normas sobre as políticas nacionais de saúde do Sistema Único de Saúde (SUS). [Internet]. [acessado 2020 maio 4]. Disponível em: https://bvsms.saude. gov.br/bvs/saudelegis/gm/2017/MatrizesConsolidacao/Matriz-2-Politicas.html

10. Bermudez JAZ, Oliveira MA, Chaves GC. Novos medicamentos: quem poderá pagar? Cad Saude Publica 2016; 32 (Supl. 2):e00025215.

11. Caetano R, Rodrigues PHA, Corrêa MCV, Villardi P, Osorio-de-Castro CGS. O caso do eculizumabe: judicialização e compras pelo Ministério da Saúde. Rev Saude Publica 2020; 54:22.

12. Wahlster P, Scahill S, Lu CY, Babar ZUD. Barriers to access and use oh high cost medicines: a review. Heal Policy Technol 2015; 4:191-214.

13. Hasan SS, Lu CY. Access to High Cost Medicines: An Overview. Chapter 1. Elsevier Inc; 2018.

14. Rover MRM, Vargas-Pelaez CM, Rocha Farias M, Nair Leite S. Acceso a medicamentos de alto precio en Brasil: la perspectiva de médicos, farmacéuticos y usuarios. Gac Sanit 2016; 30(2):110-116. 
17. Brasil. Ministério da Saúde (MS). Componente Especializado da Assistência Farmacêutica: inovação para a garantia do acesso a medicamentos no SUS [Internet]. $1^{\circ}$ ed. Brasilia: MS; 2014 [acessado 2015 nov 5]. Disponível em: http://portalsaude.saude.gov.br/ images/pdf/2014/dezembro/16/livro-2-completo-para-site.pdf

18. Brasil. Lei $\mathrm{n}^{\circ} 13.255$, de 14 de janeiro de 2016. Estima a receita e fixa a despesa da União para o exercício financeiro de 2016 [Internet]. [acessado $2020 \mathrm{abr}$ 25]. Disponível em: http://www.planalto.gov.br/ccivil_03/_Ato2015-2018/2016/Lei/L13255.htm

19. Brasil. Instituto Brasileiro de Geografia e Estatística (IBGE).Estados@ [Internet]. 2014 [acessado 2014 nov 18]. Disponível em: http://www.ibge.gov.br/estadosat/perfil.php

20. Brasil. Instituto Brasileiro de Geografia e Estatística (IBGE). Índice de Desenvolvimento Humano Municipal - IDHM. Brasília: IBGE; 2010. [acessado 2014 nov 18]. Disponível em: http://www.ibge.gov.br/estadosat/temas.php?sigla $=\&$ tema $=$ idhm

21. Brasil. Ministério da Saúde (MS). Índice de Desempenho do Sistema Único de Saúde (IDSUS). Brasília: MS; 2011 [Internet]. [acessado 2014 nov 18]. Disponível em: http://idsus.saude.gov.br/mapas.html

22. Fundação Oswaldo Cruz (Fiocruz). Pesquisa nacional de saúde. [página de Internet]. [acessado 2015 nov 15]. Disponível em: http://www.icict. fiocruz.br/sites/ www.icict.fiocruz.br/files/PNS\%20 Vol\%202.pdf

23. Brasil. Ministério da Saúde (MS). Banco de dados do Sistema Único de Saúde - DATASUS. Brasília: MS; 2016. [acessado 2016 maio 26]. Disponível em: http:// www2.datasus.gov.br/DATASUS/index.php

24. Brasil. Ministério da Saúde (MS). Portaria n ${ }^{\circ} 122$, de 31 de janeiro de 2013. Aprova o repasse de recursos para Estados e Distrito Federal, a título de financiamento, referente a janeiro, fevereiro e março de 2013, para aquisição de medicamentos do Componente Especializado da Assistência Farmacêutica, conforme Tabela de Procedimentos, Medicamentos, Órteses, Próteses e Materiais Especiais do Sistema Único de Saúde. Brasília: MS; 2013. [acessado 2016 maio 26]. Disponível em: http://bvsms.saude.gov.br/bvs/saudelegis/gm/2013/ prt0122_31_01_2013.html

25. Brasil. Ministério da Saúde (MS). Portaria $\mathrm{n}^{\circ} 757$, de 6 de maio de 2013. Aprova o repasse de recursos para Estados e Distrito Federal, a título de financiamento, referente a abril, maio e junho de 2013, para aquisição de medicamentos do Componente Especializado da Assistência Farmacêutica, conforme Tabela de Procedimentos, Medicamentos, Órteses, Próteses e Materiais Especiais do Sistema Único de Saúde. Brasília: MS; 2013. [acessado maio 6, 2013. [acessado 2016 maio 26]. Disponível em: http://bvsms.saude.gov.br/bvs/saudelegis/gm/2013/prt0757_06_05_2013.html

26. Brasil. Ministério da Saúde (MS). Portaria $\mathrm{n}^{\circ} 1.659$, de 8 de agosto de 2013. Aprova o repasse de recursos para Estados e Distrito Federal, a título de financiamento, referente a julho, agosto e setembro de 2013, para aquisição de medicamentos do Componente Especializado da Assistência Farmacêutica conforme Tabela de Procedimentos, Medicamentos, Órteses, Próteses e Materiais Especiais do Sistema Único de Saúde. Brasília: MS; 2013. [acessado 2016 maio 26]. Disponível em: http://bvsms.saude.gov.br/bvs/saudelegis/gm/2013/ prt1659_08_08_2013.html

27. Brasil. Ministério da Saúde (MS). Portaria no 2.701 , de 11 de novembro de 2013. Aprova o repasse de recursos para os Estados e Distrito Federal, a título de financiamento, referente a outubro, novembro e dezembro de 2013, para aquisição de medicamentos do Componente Especializado da Assistência Farmacêutica conforme Tabela de Procedimentos, Medicamentos, Órteses, Próteses e Materiais Especiais do Sistema Único de Saúde. Brasília: MS; 2013. [acessado 2016 maio 26]. Disponível em: http://bvsms.saude.gov.br/bvs/saudelegis/ gm/2013/prt2701_11_11_2013.html

28. Pope C, Ziebland S, Mays N. Analysing qualitative data. BMJ 2000; 320(7227):114-116.

29. Brasil. Ministério da Saúde (MS). Pesquisa Nacional sobre Acesso, Utilização e promoção do uso racional de Medicamentos no Brasil, PNAUM: Primeiros Resultados Brasília: MS; 2015. [acessado 2016 maio]. Disponível em: http://www.ufrgs.br/pnaum/projeto-1/ arquivos/pnaum-primeiros-resultados

30. Sheikh K, Gilson L, Agyepong IA, Hanson K, Ssengooba F, Bennett S. Building the Field of Health Policy and Systems Research: Framing the Questions. PLoS Med 2011; 8(8):e1001073.

31. Paina L, Peters DH. Understanding pathways for scaling up health services through the lens of complex adaptive systems. Health Policy Plan 2012;27(5):365373.

32. Bigdeli M, Jacobs B, Tomson G, Laing R, Ghaffar A, Dujardin B, Damme WV. Access to medicines from a health system perspective. Health Policy Plan 2013; 28(7):692-704.

33. Luz TCB, Loyola Filho AI, Lima-Costa MF. Estudo de base populacional da subutilização de medicamentos por motivos financeiros entre idosos na Região Metropolitana de Belo Horizonte, Minas Gerais, Brasil. Cad Saude Publica 2009; 25(7):1578-1586.

34. Penchansky R, Thomas JW. The concept of access: definition and relationship to consumer satisfaction. Med Care 1981;19(2):127-140.

35. Silva AAS, Costa SMC. A descentralização do componente especializado da assistência farmacêutica na $15^{\text {a }}$ região de saúde do estado do Ceará. RBFHSS 2015; 6(1):37-40

36. Garcia-Subirats I, Vargas I, Mogollón-Pérez AS, Paepe P, Silva MRF, Unger JP, Vázquez ML. Barriers in access to healthcare in countries with different health systems. A cross-sectional study in municipalities of central Colombia and north-eastern Brazil. Soc Sci Med 2014; 106:204-213.

37. Lima-Dellamora EC, Caetano R, Osorio-de-Castro CGS. Dispensing specialized component medicines in areas of the State of Rio de Janeiro. Cien Saude Colet 2012; 17(9):2387-2396. 
38. Buendgens FB, Blatt CR, Marasciulo ACE, Leite SN, Farias MR. Cost analysis of treatment for severe rheumatoid arthritis in a city in southern Brazil. Cad Saude Publica 2013; 29:s81-91.

39. Gerlack LF, Karnikowski MGO, Areda CA, Galato D, Oliveira AG, Álvares J, Leite SN, Costa EA, Guibu IA, Soeiro OM, Costa KS, Guerra Junior AA, Acurcio FA. Gestão da assistência farmacêutica na atenção primária no Brasil. Rev Saude Publica 2017; 51(Supl. 2):15s.

40. Carvalho MN, Álvares J, Costa KS, Guerra Junior AA, Acurcio FA, Costa EA, Guibu IA, Soeiro OM, Karnikowski MGO, Leite SN. Força de trabalho na assistência farmacêutica da atenção básica do SUS, Brasil. Rev Saude Publica 2017; 51(Supl. 2):16s.

41. Leite SN, Manzini F, Álvares J, Guerra Junior AA, CostaEA, Acurcio FA, Guibu IA, Costa KS, Karnikowski MGO, Soeiro OM, Farias MR. Infraestrutura das farmácias da atenção básica no Sistema Único de Saúde: Análise dos dados da PNAUM-Serviços. Rev Saude Publica 2017; 51(Supl. 2):13s.

42. Vargas I, Mogollón-Pérez AS, Unger J-P, da-Silva MRF, Paepe PD, Vázquez M-L. Regional-based Integrated Healthcare Network policy in Brazil: from formulation to practice. Health Policy Plan 2015; 30(6):705-717.

43. Nascimento RCRM, Álvares J, Guerra Junior AA, Gomes IC, Costa EA, Leite SN, Costa KS, Soeiro OM, Guibu IA, Karnikowski MGO, Acurcio FA. Disponibilidade de medicamentos essenciais na atenção primária do Sistema Único de Saúde. Rev Saude Publica 2017; 51(Supl. 2):10s.

44. Kuschnir R, Chorny AH. Health care networks: contextualizing the debate. Cien Amp Saude Colet 2010; 15(5):2307-2316.

45. Rover MRM. Avaliação da capacidade de gestão do componente especializado da assistência farmacêutica em Santa Catarina [tese]. Florianópolis: Universidade Federal de Santa Catarina; 2016.

46. Tobar F, Drake I, Martich E. Alternativas para la adopción de políticas centradas en el acceso a medicamentos. Rev Panam Salud Publica 2012; 32(6):457-463.

47. Brasil. Ministério da Saúde (MS). Portaria $\mathrm{n}^{\circ} 34$, de 6 de agosto de 2013. Torna pública a decisão de não incorporar o medicamento brometo de tiotrópio para o tratamento da doença pulmonar obstrutiva crônica no Sistema Único de Saúde (SUS). Brasília: MS; 2013. [acessado 2020 abr 25]. Disponível em: http://bvsms.saude.gov.br/bvs/saudelegis/sctie/2013/ prt0036_06_08_2013.html

48. Brasil. Ministério da Saúde (MS). Secretaria de Ciência, Tecnologia e Insumos Estratégicos Departamento de Gestão e Incorporação de Tecnologias em Saúde Coordenação de Avaliação e Monitoramento de Tecnologias. Relatório de Recomendação: Insulinas análogas de ação prolongada para o tratamento de diabetes mellitus tipo II. Brasília: MS; 2018. [acessado 2020 abr 25]. Disponível em: http://conitec.gov.br/images/ Consultas/Relatorios/2018/Relatorio_InsulinasAnalogas_AcaoProlongada_DM2_CP80_2018.pdf
49. Brasil. Ministério da Saúde (MS). Secretaria de Ciência, Tecnologia e Insumos Estratégicos. Relatório de Recomendação: Ranibizumabe para Degeneração Macular Relacionada à Idade. Brasília: MS; 2015. [acessado 2020 abr 25]. Disponível em: http://conitec. gov.br/images/Relatorios/2015/Relatorio_Ranibizumabe_DMRI_final.pdf

50. Figueiredo TA, Pepe VLE, Osorio-de-Castro CGS. A sanitary focus on medicines lawsuit. Physis 2010; 20(1):101-118.

51. Programa de Apoio às Secretarias Estaduais de Saúde (Pases) - Projeto (Proadi-SUS): Cuidado Farmacêutico no Componente Especializado da Assistência Farmacêutica. [acessado 2020 abr 25]. Disponível em: http:// www.conass.org.br/programa-de-apoio-as-secretarias-estaduais-de-saude/

52. García-Altés A, Zonco L, Borrell C, Plasència A. Measuring the performance of health care services: a review of international experiences and their application to urban contexts. Gac Sanit 2006; 20(4):316-324.

53. Arrais PSD, Brito LL, Barreto ML, Coelho HLL. Prevalência e fatores determinantes do consumo de medicamentos no Município de Fortaleza, Ceará, Brasil. Cad Saude Publica 2005; 21(6):1737-1746.

54. Fritzen JS, Motter FR, Paniz VMV. Acesso regular e adesão a medicamentos do componente especializado assistência farmacêutica. Rev Saude Publica 2017; 51:109.

55. Brasil. Ministério da Saúde (MS). Portaria no 13, de 6 de janeiro de 2020. Altera o Título IV do Anexo XXVIII da Portaria de Consolidação no 2/GM/MS, de 28 de setembro de 2017, que dispõe sobre as regras de financiamento e execução do Componente Especializado da Assistência Farmacêutica no âmbito do Sistema Único de Saúde (SUS). Brasília: MS; 2020. [acessado 2020 abr 25]. Disponível em: http://www.in.gov.br/web/dou/-/ portaria-n-13-de-6-de-janeiro-de-2020-237059261.

Artigo apresentado em 07/05/2020

Aprovado em 26/07/2020

Versão final apresentada em 28/07/2020

Editores-chefes: Romeu Gomes, Antônio Augusto Moura da Silva 